\title{
Accessing the online evidence: a guide to key sources of research information on clinical and cost effectiveness
}

\author{
J Glanville, P Wilson, R Richardson
}

Qual Saf Health Care 2003;12:229-231

Advice on how to access the best available online sources of research evidence on clinical and cost effectiveness published in three recent issues of Effectiveness Matters is reviewed.

$\mathrm{T}$ his article is based on three recent issues of Effectiveness Matters that provided advice on how to access the best available online sources of research evidence on clinical and cost effectiveness. $^{1-3}$

In the UK, healthcare professionals are being encouraged to ensure that their practice is based on the best evidence from well conducted research. Clinical governance in the NHS has given all health organisations, including primary care groups, a statutory duty to seek quality improvements in the health care delivered by their organisation. ${ }^{45}$ Making quality improvements requires the successful identification, assessment, and application of high quality research evidence about costs and effectiveness by those making clinical, managerial, and policy decisions.

High quality research evidence is increasingly being made available via the internet. This paper describes how to access key online sources of research evidence on clinical and cost effectiveness (see box 1). More detail on how to search these resources is available elsewhere. ${ }^{67}$

\section{QUALITY ASSESSED EFFECTIVENESS INFORMATION}

Research evidence is proliferating. Busy healthcare professionals can benefit from resources that have identified best evidence and present it in easy to use formats.

Systematic reviews are useful tools for busy decision makers because they identify, appraise, and synthesise the available research evidence on a particular topic. Many thousands of systematic reviews relevant to health care have been published. However, they can be difficult to locate $^{8}$ and their quality can be variable. ${ }^{9}$

See end of article for authors' affiliations

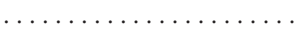

Correspondence to: Julie Glanville, Centre for Reviews \& Dissemination, University of York, York YO10 5DD, UK;

jmgl@york.ac.uk

\section{Overviews of the research evidence}

There are now major publications that summarise the research evidence across broad subject areas. These are usually available both in a paper format and on the internet. Clinical Evidence is a monthly updated directory of evidence on the effects of common clinical interventions published by the BMJ Publishing Group. The Health Evidence Bulletins Wales are evidence based summaries of treatments in broad disease areas such as mental health, cancers, and injury prevention. Effective Health Care bulletins summarise the best evidence from published research. Other collections of reviews, technology assessments, and appraisals can be found at the websites of the National Institute for Clinical Excellence (NICE) and the National Coordinating Centre for Health Technology Assessment (NCCHTA).

In the UK a major national initiative has been the development of the online National electronic Library for Health (NeLH), a "high performance knowledge base to support effective health care" ${ }^{\prime 10}$ The NeLH aims to provide health professionals with access to the best current "know how" and "knowledge" and includes access to all of the key resources mentioned in this paper.

\section{The Cochrane Library}

The Cochrane Library is an electronic publication containing a regularly updated collection of research databases. One of these, the Cochrane Database of Systematic Reviews (CDSR), is a unique cumulative collection of full text systematic reviews. These reviews are valuable, not only for their rigorous methodology, but also because they are periodically updated as new research evidence is published and in response to valid criticisms.

The Cochrane Library also includes the Database of Abstracts of Reviews of Effects (DARE). This is a monthly updated database of abstracts of quality assessed systematic reviews of the effects of healthcare interventions. Systematic reviews are identified by hand searching key medical and related journals, regular searching of bibliographic databases, and by scanning "grey" literature such as conference abstracts, reports, and theses. These reviews are then assessed for inclusion against predetermined criteria. For reviews that meet these criteria, detailed abstracts are written describing the review methods, results and conclusions, along with a critical commentary on the overall quality of the review. When searching the Cochrane Library, records from DARE will automatically be included in the search results. DARE is also available free of charge on the internet.

If reviews are unavailable for a specific topic, well conducted randomised controlled trials provide the next best level of research evidence. The Cochrane Central Register of Controlled Trials (CENTRAL) is the largest register of controlled trials in the world, and includes hand searched information from unpublished reports and conference proceedings as well as records from bibliographic databases such as MEDLINE and EMBASE. 


\section{Box 1 Key effectiveness resources}

This list is indicative and oriented to the UK. Further resources are listed on the Centre for Reviews and Dissemination (CRD) website (http://www.york.ac.uk/ inst/crd/em51_app 1.htm) or can be obtained by contacting CRD.

\section{Gateways and indexes}

- National Electronic Library for Health (NeLH): http:// www.nelh.nhs.uk/

- Netting the evidence: http://www.nettingtheevidence.org.uk/

- SumSEARCH: http://SUMSearch.uthscsa.edu/searchform4. $\mathrm{htm}$

- TRIP database: http://www.tripdatabase.com/

\section{Reviews of effectiveness}

- Cochrane Library: http://www.cochrane.org and http:// www.nelh.nhs.uk

- DARE (Database of Abstracts of Reviews of Effects): http:// nhscrd.york.ac.uk/darehp.htm

- HTA (Health Technology Assessment) database: hHtp:// nhscrd.york.ac.uk/htahp.htm

- National Coordinating Centre for Health Technology Assessment (NCCHTA): http://www.hta.nhsweb.nhs.uk/

- National Institute for Clinical Excellence (NICE): hHtp:// www.nice.org.uk/nice-web/

\section{Evidence overviews}

- Bandolier: http://www.jr2.ox.ac.uk/bandolier/

- Clinical Evidence: http://www.clinicalevidence.com/

- Effective Health Care bulletins: http://www.york.ac.uk/ inst/crd/ehcb.htm

- Health Evidence Bulletins Wales: http://hebw.uwcm. ac.uk/

\section{Other resources}

- NHSEED (NHS Economic Evaluation Database): http:// agatha.york.ac.uk/nhsdhp.htm

- Ovid Evidence-Based Medicine Reviews: http:// www.ovid.com/

- PubMed: http://www.ncbi.nlm.nih.gov/entrez/query.fcgi

- Search filter collections: http://hebw.uwcm.ac.uk/ methodology/appendix2.htm

\section{Information on cost effectiveness}

Other databases included in the Cochrane Library are the NHS Economic Evaluation Database and the Health Technology Assessment (HTA) database. Although there is a growing literature evaluating the cost effectiveness of healthcare interventions, these studies may be difficult to identify and appraise. This is especially true for readers who are not familiar with the different methods of economic evaluation. The interpretation of economic evaluations may also be limited by the reader's knowledge of what constitutes good research.

Easy access to information on the cost effectiveness of interventions is provided by the NHS Economic Evaluation Database (NHSEED). NHSEED is a collection of critical assessments of published economic evaluations of healthcare interventions which is updated monthly. Economic evaluations are identified from the large number of published research studies that deal with the economics of health care and health technology assessment. Each evaluation is assessed by a health economist who provides a structured abstract of the original study to help decision makers to decide on its quality and relevance to their own situations. To ensure that the information is of maximum benefit to the intended user, abstracts normally describe the clinical effectiveness information on which the economic evidence is based, as well as providing a detailed breakdown of the key components of the economic evaluation. A commentary summarises the overall reliability of the paper and presents any practical implications for the NHS.

\section{Gateways and indexes to effectiveness resources}

Clinical effectiveness publications appear in a range of formats and this can make them difficult to locate. Searchable indexes are therefore highly important, even given time lags between publication and appearance in an index. The Turning Research into Practice (TRIP) database is a free internet search service that covers a wide range of UK and US clinical effectiveness resources and evidence based guidelines. It indexes many of the resources mentioned in this document and can be searched by topic. There is a link to the TRIP database from the NeLH.

Netting the evidence is another useful internet tool that provides a route into the many available resources that can be used to practise and inform evidence based health care.

\section{Journal clubs and critical appraisal journals}

There are further routes to assessed and value added evidence publications. Evidence-Based Medicine, Evidence-Based Nursing, and other journals in the Evidence-Based series assess and comment on high quality published reviews and primary studies. Bandolier highlights new research evidence and conducts critical appraisals of research together with brief reviews.

\section{Bibliographic databases}

If the focused resources described above do not provide reviews or appraisals on a topic of interest, it may be desirable to search other databases. Large databases such as MEDLINE or PsycINFO (a major database for psychological research) provide access to a wealth of unassessed research evidence. However, further steps will be required to obtain and assess the full texts of records retrieved. Advice on the local availability of these resources and how to search them should be obtainable from health libraries.

The SumSearch service in the USA gives searchers the opportunity to carry out searches across a range of resources including MEDLINE, DARE and selected journals, and presents the results in a useful hierarchy including "broad discussions", systematic reviews, and trials. A link to SumSearch is available from the NeLH.

A number of predesigned systematic review search filters (collections of search terms) are available for MEDLINE to which a searcher need only add specific subject terms. Some versions of MEDLINE such as PubMed Clinical Queries offer built-in filters that can focus a search onto treatment, prognosis, and other options. Searchers should be aware that filters retrieve research of varying quality and that quality assessment of the results remains the task of the searcher.

\section{INFORMATION SUPPORT}

Many health professionals may not have the time, resources, or skills to access information services effectively. However, there are often specialists trained in searching who can help. ${ }^{11-13}$ Many health professionals have access to librarians or other information support services. Some health libraries and information services will carry out searches on behalf of health professionals. Contacting a local healthcare librarian will help to identify which resources are available locally and whether assistance is available in terms of training or performing searches. When approaching services for information on a specific effectiveness topic, it is always helpful to prepare as clear a question as possible in advance. ${ }^{14}$

There are also specialist information services, such as the CRD Information Service, which have been funded to provide assistance in obtaining information from the best available research evidence. This service can be accessed by telephone (01904 433707) or email (crd-info@york.ac.uk), from 09.00 to 05.15 hours Monday to Friday. These services vary in their 
remit, the extent of their assistance, and geographical coverage, but can provide valuable help to busy health professionals. A selected list of UK based specialist information services that focus on searching for research evidence is available from the CRD website (http://www.york.ac.uk/inst/crd/ em51_appl.htm).

\section{SUMMARY}

High quality effectiveness information to inform healthcare practice is increasingly available online. The best strategy to locate this information is to search the key focused resources described in this paper or to use any available support services offered by librarians, information professionals, and appropriate trainers.

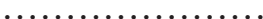

\section{Authors' affiliations}

J Glanville, P Wilson, R Richardson, Centre for Reviews \&

Dissemination, University of York, York YO10 5DD, UK

\section{REFERENCES}

1 NHS Centre for Reviews and Dissemination. Accessing the evidence on clinical effectiveness. Effectiveness Matters 2001;5(1).

2 NHS Centre for Reviews and Dissemination. Cost-effectiveness matters: providing the NHS Economic Evaluation Database (NHS EED). Effectiveness Matters 2002;6(1).
3 NHS Centre for Reviews and Dissemination. The Database of Abstracts of Reviews of Effects (DARE). Effectiveness Matters 2002;6(2). 4 Scally G, Donaldson L. The NHS's 50th anniversary. Clinical governance and the drive for quality improvement in the new NHS in England. BM 1998;317:61-5.

5 Secretary of State for Health. A first class service: quality in the new NHS. London: The Stationery Office, 1997.

6 Glanville J, Lefebvre C. Identifying systematic reviews: key resources. Evidence-based Mental Health 2000;3:68-9.

7 Glanville J, Lefebvre C. Identifying systematic reviews: key resources. Evidence-based Medicine 2000;5:68-9.

8 Boynton J, Glanville J, McDaid D, et al. Identifying systematic reviews in MEDLINE: developing an objective approach to search strategy design. $J$ Inform Sci 1998;24: 137-57

9 Petticrew M, Song F, Wilson P, et al. Quality-assessed reviews of health care interventions and the database of abstracts of reviews of effectiveness (DARE). Int J Technol Assess Health Care 1999;15:671-8.

10 Toth B, Muir Gray DA, Fraser V, et al. National electronic Library for Health. Progress and prospects. Health Libraries Rev 2000;17:46-50.

11 Fuller A, Ketchell DS, Tarczy-Hornoch P, et al. Quality filtering of the clinical literature by librarians and physicians. Bull Med Libr Assoc 1993;81:38-43.

12 Erickson S, Warner E. The impact of individual tutorial sessions on MEDLINE use among obstetrics and gynaecology residents in an academic training programme: a randomized trial. Med Educ 1998;32:269-73

13 Rosenberg W, Deeks J, Lusher A, et al. Improving searching skills and evidence retrieval. J R Coll Phys London 1998;32:6557-63.

14 Centre for Evidence-based Medicine. Focusing clinical questions. Oxford: Centre for Evidence-based Medicine, 2000 (http:// www.cebm.net/focus_quest.asp). 